\title{
Resiliência, suporte social e prática esportiva: relações e possibilidades de intervenção social
}

\author{
Resilience, social support and sports practice: relations and social intervention \\ possibilities
}

Paulo Castelar Perim ${ }^{1}$

Nuno Corte-Real ${ }^{2}$

Cláudia Dias ${ }^{3}$

Melissa Parker ${ }^{4}$

Lidio de Souza (in memoriam)

Antonio Manuel da Fonseca ${ }^{5}$

\begin{abstract}
RESUMO: Embora nos últimos anos tenha vindo a aumentar o número de pesquisas que examinam a resiliência e o suporte social no contexto do esporte de competição, são ainda necessários estudos que examinem estas variáveis em contexto não competitivo, junto de crianças e jovens. Esta pesquisa teve por objetivo estudar a prática esportiva, a resiliência e o suporte social junto de 1052 com idades entre os 12 e os 20 anos $(15,53 \pm 1,68)$, estudantes de escolas públicas brasileiras. Inicialmente, examinaram-se as relações entre o sexo e a idade com a prática esportiva, resiliência e suporte social. Em seguida, averiguou-se a existência de diferenças nos níveis de resiliência e suporte social em função de diferentes níveis de prática esportiva. Os participantes preencheram uma bateria de questionários. Os resultados revelaram que os alunos mais novos praticavam esporte com mais frequência que as moças e os mais velhos. Não foram encontradas diferenças entre sexos nos níveis de resiliência, mas as moças estavam mais satisfeitas com o suporte social do que os rapazes. Os alunos no início da adolescência eram menos resilientes e estavam menos satisfeitos com o seu suporte social do que os jovens adultos e os alunos a meio da adolescência, respetivamente. Os resultados apontam ainda para uma relação significativa entre a prática esportiva e a resiliência, bem como entre a prática esportiva e o grau de satisfação dos sujeitos com suas redes de suporte social, independentemente do número de sujeitos que compunham esta rede.
\end{abstract}

Palavras-chave: resiliência; psicologia do esporte; redes de suporte social; avaliação psicológica; intervenção social.

ABSTRACT: Although in recent years the number of research examining resilience and social support in competitive sports context has increased, further studies are needed to examine these variables in non-competitive contexts, with children and young people. This research aimed to study sports practice, resilience and social support in 1052 Brazilian students from public schools, aged 12 and 20 years old (15.53 \pm 1.68$)$. Initially, we examined the relationship between sex and age with sports practice, resilience and social support. Afterwards, we examined differences in resilience and social support across different levels of sports practice. Participants completed a pack of questionnaires. The results revealed that the boys and younger students practiced sport

\footnotetext{
${ }^{1}$ Doutor em Ciências do Desporto; Professor Adjunto do Departamento de Psicologia da Universidade Federal do Espírito Santo - Vitória, ES, Brasil. E-mail: pcastelar@hotmail.com.

2 Doutor em Ciências do Desporto; Professor da Faculdade de Desporto (CIFI2D) da Universidade do Porto - Porto, Portugal.

${ }^{3}$ Doutora em Psicologia; Professora da Faculdade de Desporto (CIFI2D) da Universidade do Porto - Porto, Portugal.

${ }^{4}$ Doutora em Ciências do Desporto; Professora no Physical Education \& Sport Sciences Department - University of Limerick, Limerick.

${ }^{5}$ Doutor em Ciências do Desporto; Professor Catedrático da Faculdade de Desporto (CIFI2D) da Universidade do Porto Porto, Portugal.
} 
more often than girls and older students. No differences were found between sexes in resilience levels, but girls were more satisfied with their perceived social support than boys. Students in their early teens were less resilient and were less satisfied with the social support they perceived than young adults and students in their mid-teens, respectively. The results also indicated a significant relationship between sports practice and resilience, as well as between sports and the level of satisfaction with the social support network, regardless of the number of subjects that made up that network.

Keywords: resilience; sport psychology; social support networks; psychological evaluation; social intervention.

\section{Introdução}

Atualmente, os múltiplos benefícios interligados a uma atividade física e/ ou esportiva regular e sistemática são claramente reconhecidos e inegáveis. Com efeito, numerosas pesquisas têm sido desenvolvidas buscando descortinar as relações entre a prática de atividade física (e da prática esportiva) em variados aspectos da realidade humana, incluindo o autoconceito (Fox \& Corbin, 1989; Marsh \& Craven, 1997; Marsh \& Perry, 2005), os estilos de vida saudáveis (Corte-Real, 2006), o bem estar subjetivo (Balaguer, 2000; Standage, Duda, \& Pensgaard, 2005), ou o desenvolvimento positivo dos jovens (Hellison, 1985; Hellison \& Wash, 2002; Regueiras, 2012). De uma forma geral, os resultados desses estudos acentuam o potencial da atividade física e do esporte, demonstrando que a sua prática traz benefícios não só a nível físico, mas também contribui, de forma singular, para o desenvolvimento psicossocial dos indivíduos nela envolvidos (Balaguer \& Castillo, 2002; Dias et al., 2008).

Por outro lado, na última década, tanto as Nações Unidas, como vários governos e entidades não-governamentais, bem como associações esportivas por várias partes do mundo, têm adotado programas esportivos para prevenir e tratar diversos problemas de saúde e de desenvolvimento pessoal e social de crianças e jovens. Estes programas têm sido usados para atender e ajudar pessoas que enfrentaram e/ ou enfrentam dificuldades em diversas áreas, como pandemias, problemas sociais (buscando integrar crianças de rua), pobreza e desastres, objetivando ajudar a restabelecer o seu equilíbrio psicológico e social (Henley, Schweizer, Gara, \& Vetter 2008). Na sua base estão evidências de que o esporte fornece uma oportunidade ímpar para que sejam estabelecidas relações afetivas e laços saudáveis entre grupos de pares de crianças e jovens, assim como com adultos significativos (Brodkin \& Weiss, 1990; Weiss \& Petlichkoff, 1989). Parece ser que, mesmo quando tardios estes laços são bastante eficazes para ajudar e até mesmo interceptar trajetórias de vida que estão se desenvolvendo de forma negativa, podendo contribuir de forma eficaz para a prevenção contra futuros enfrentamentos de riscos (Luthar \& Cicchetti, 2000; Rutter, 2006).

Uma área que, nos últimos anos, tem sido particularmente estudada em relação com estes programas é a resiliência. A resiliência é um conceito ainda em construção que, inicialmente, era considerado uma característica intrínseca dos sujeitos, uma característica que seria inata. Ou seja, os sujeitos nasceriam resilientes e as circunstâncias da vida determinariam o seu desenvolvimento, tanto positiva como negativamente. Wagnild (2009) considerava que a manifestação da resiliência seria um processo que poderia ser despertado, ou não, dependendo das circunstâncias da vida. Para este autor, o caráter da resiliência não é um processo, mas sim uma qualidade inata de certas pessoas.

Todavia, há cerca de duas décadas, diversos autores começaram a considerar a resiliência o equivalente humano ao que se estudava na Física, ou seja, à propriedade que alguns elementos possuem de retornar ao estado anterior após terem sido submetidos a 
qualquer elemento de pressão. Entretanto, no que se refere à resiliência humana, esta se manifestaria como a propriedade de minimizar ou dominar os efeitos nocivos da adversidade como resposta ao risco (Zomignani, 2002). Desta forma, é preciso muito mais do que sobreviver ao risco (Ralha-Simões, 2001). Torna-se necessário que o sujeito enfrente o risco, sendo afetado por ele, mas assumindo uma resposta positiva que the permita ultrapassá-lo, aprendendo com ele e retirando ganhos desta experiência (Rutter, 2006; Yunes \& Szymanski, 2001). Assim, com o surgimento de pesquisas e estudos que demonstraram que a resiliência se trata de um processo que possui uma certa relatividade, dado que vai variar de acordo com o sujeito e as circunstâncias onde ela vai ocorrer (Luthar, Cichetti, \& Becker, 2000; Rutter, 2006; Yunes, \& Szymanski, 2001), deixou-se de se falar em sujeito resiliente para se falar num processo de resiliência. Neste contexto, estudos que centralizavam suas pesquisas nas características pessoais das 'crianças resilientes' passaram a discutir a resiliência como um processo onde se encontram, para além dos atributos da criança, os aspectos familiares e sociais (Masten \& Garmezy, 1985; Werner \& Smith, 1992).

Deste modo, pode-se afirmar que a resiliência não é unicamente um traço de personalidade ou um atributo fixo de determinadas pessoas, mas antes algo construído dentro do processo de interação social, onde estão em jogo mecanismos de risco e proteção, as respostas individuais, as contribuições relativas aos vínculos familiares e as redes de apoio social (Cyrulnik, 2001; Rutter, 2006). Em suma, a resiliência pode ser entendida como o processo que possibilita transformar uma situação traumática e dolorosa numa possibilidade de aprendizagem e de crescimento, ou, ainda melhor, o processo que facilita o combate da adversidade percebida e causadora de danos, possibilitando ao sujeito sair dela fortalecido (Grotberg, 2001). Ou, nas palavras de Assis, Avanci, Pesce e Deslandes (2006), resiliência significa a capacidade de "encontrar forças para transformar intempéries em perspectivas" (p. 19).

Os estudos acerca da resiliência têm proliferado em várias partes do mundo. Uma imagem clara do lugar que este construto tem vindo a assumir no interesse dos pesquisadores foi dada por Wagnild (2009), que verificou que, só numa breve revisão, e utilizando apenas uma base de dados (PubMed), a palavra 'resiliência' aparecia cerca de 11 vezes entre 1977 e 1987, e mais de 600 vezes nas duas décadas seguintes; além disso, apenas entre Janeiro e Abril de 2007, foi referida em 50 estudos. Claramente, o interesse na resiliência está em crescimento e abrange aspectos cada vez mais específicos da vida e do quotidiano humanos. Só na base de dados do Scielo-Brasil, aparecem atualmente, em 2015, 227 artigos com a palavra resiliência. As temáticas são muito abrangentes e incluem, entre outras, as condições adversas da vida de certas populações (desvantagens socioeconômicas) e os riscos a ela associados (Garmezy, 1991; Werner \& Smith, 1992), doenças mentais (Masten \& Coatsworth, 1998), maus-tratos de crianças (Beeghly \& Cicchetti, 1994; Cicchetti \& Rogosch, 1997), pobreza urbana e violência na comunidade (Richter \& Martinez, 1993), doenças crônicas (Wells \& Schwebel, 1987), ou acontecimentos de vida catastróficos (O'Dougherty-Wright, Masten, Northwood, \& Hubbard, 1997).

Nos estudos que buscaram correlacionar a resiliência com o sexo, alguns têm indicado vantagens para os sujeitos do sexo masculino (Hunter \& Chandler, 1999; Leppert, Gunzelmann, Schumacher, Strauss, \& Brahler, 2005). No entanto, uma análise de 12 estudos que tinham utilizado a Resilience Scale concluiu que apenas dois exibiam diferenças entre homens e mulheres e que em nenhum havia diferenças relacionadas com a idade (Wagnild, 2009). Entretanto, num estudo realizado com o mesmo instrumento na Suécia, Lundman, 
Strandberg, Eisemann, Gustafson, e Brulin (2007) encontraram relações positivas entre a idade e o desenvolvimento da resiliência. Para explicar os dados encontrados, que se diferenciavam de outros estudos, os autores chamaram a atenção para o fato do seu estudo abranger um elevado número de participantes (cerca de 1990, entre profissionais da saúde e idosos), com idades bastante variadas. Isto não teria ocorrido em outras pesquisas, onde a variabilidade das idades dos sujeitos era menor. Lundman et al. (2007) argumentam ainda que a resiliência deveria ser considerada como um processo, o qual, não sendo estático, poderia mudar ao longo da vida, o que explicaria também os seus resultados.

Por outro lado, parece ser consensual que a prática esportiva está associada à resiliência, especialmente quando nos referimos ao esporte de competição. De um modo geral, os atletas, independentemente de estarem no nível amador ou no profissional, em competição de alto nível ou não, costumam traçar objetivos que, geralmente, representam grandes desafios que os levam a enfrentar vários obstáculos em suas carreiras (por exemplo: derrotas severas, lesões). Mesmo assim, continuam a competir, enfrentando de forma corajosa estes riscos que vão aparecendo. Ao enfrentá-los, se tornam vencedores, demonstrando que a prática esportiva é, por excelência, um espaço de produção de comportamentos resilientes (Galli \& Vealey, 2008; Miller \& Kerr, 2002). O fato de não sucumbirem a estes percalços e conseguirem sair deles renovados e vitoriosos poderá, eventualmente, decorrer de suas capacidades/ competências de apresentarem comportamentos resilientes.

Ao nível da investigação, as pesquisas que estudaram a resiliência no esporte adotaram a ideia expressa por Richardson (2002), que distingue três momentos no desenvolvimento do conceito de resiliência: a primeira, segunda e terceira onda. A primeira onda caracterizou-se pelo estudo das qualidades internas e externas das pessoas resilientes, ou seja, das pessoas que tinham sucesso social e pessoal apesar de estarem na presença de adversidades. A segunda onda teria objetivos diferenciados: ao invés de tentar compreender as qualidades dos atletas resilientes, o foco seria centrado no processo. Por outras palavras, iria além da identificação das qualidades do atleta resiliente e procuraria estudar o processo e de que forma estes atributos e qualidades foram adquiridos (Galli \& Vealey, 2008; Richardson, 2002). Uma terceira onda centrou-se na resiliência inata, no sentido de identificar as forças motivacionais que levam os indivíduos a uma reintegração, após passarem por situações adversas. Ou seja, as pesquisas na área da psicologia do esporte seguiram a evolução que ocorreu na psicologia geral, de uma abordagem mais centrada nas pessoas, para uma abordagem mais centrado no processo.

O estudo de Galli e Vealey (2008), no qual os autores exploraram as percepções e experiências de resiliência de 10 atletas de elite que, ao longo de suas carreiras, tiveram que enfrentar várias dificuldades, apontam exatamente nesse sentido. Mais do que conhecer as características da pessoa resiliente, esta pesquisa teve como objetivo conhecer os processos que estariam subjacentes ao desenvolvimento de comportamentos resilientes por parte destes atletas. Os autores não só concluíram que a resiliência é um processo (em vez de um traço ou resultado), mas também constataram que as estratégias de enfrentamento e os ambientes e as redes de apoio social dos atletas eram determinantes na construção de comportamentos resilientes.

Fletcher e Sarkar (2012) também exploraram a resiliência junto de atletas de elite, neste caso 12 atletas olímpicos (medalha de ouro). Os autores concluíram que a resiliência era um conceito abrangente relacionado às respostas dos atletas a estressores, que incluía 
fatores como motivação, foco, confiança, personalidade positiva e suporte social, e que permitia aos atletas avaliarem os estressores como desafios em vez de problemas, bem como avaliarem o seu próprio pensamento (metacognição). Recentemente, White e Bennie (2015) investigaram as percepções de treinadores e atletas de ginástica sobre o desenvolvimento da resiliência no esporte. Treinadores e atletas acreditavam que a resiliência se desenvolve durante o treino e competição, sendo fundamental uma atmosfera de treino amigável e positiva.

Ainda no esporte de competição, Hosseini e Besharat (2010) desenvolveram um estudo com 139 jovens atletas de várias modalidades esportivas, onde investigaram a relação entre a prática esportiva, o bem-estar psicológico e a resiliência. Os resultados mostraram que a resiliência estava positivamente associada ao desempenho esportivo e ao bem-estar psicológico, e negativamente associado ao estresse psicológico. Em suas considerações, sugerem a importância do desenvolvimento de mais pesquisas que possam compreender ainda melhor esta relação, chamando a atenção para o fato de estes estudos poderem contribuir para os processos de educação que visam o desenvolvimento dos níveis de resiliência entre os atletas. Na mesma linha, Chung et al. (2013) avaliaram o impacto de um programa de treino de resiliência num grupo de atletas juniores de diferentes modalidades, contrastando-o com um programa tradicional de treino de habilidades psicológicas. Os resultados mostraram que o programa de resiliência foi eficaz na melhora geral da resiliência psicológica dos atletas, tendo tido, a esse respeito, melhores resultados do que o programa de treino de habilidades psicológicas.

Em contexto não competitivo, o esporte e programas com jogos também têm sido apontados como elementos de grande utilidade para ajudar crianças e jovens especialmente aqueles que sofreram processos severos de estresse ou traumas -, a passarem pelas experiências necessárias para aprenderem habilidades e valores sociais (aprendendo a lidar com a exclusão, a gerir suas emoções, a se autocontrolarem, e a partilharem poder, espaço e ideias) e, deste modo, desenvolverem comportamentos de resiliência (Henley et al., 2008). Em uma investigação de Sanches (2007), por exemplo, qual a autora estudou se a resiliência podia ser uma atividade potencialmente promotora de resiliência em adolescentes da Organização Fluminense de Atletismo - um projeto social que ensinava a prática desta modalidade a crianças e jovens entre os 12 e os 18 anos -, confirmou-se que o esporte favorecia, de fato, muitos aspectos essenciais para a promoção de resiliência. Especificamente, o projeto contribuiu para o fortalecimento da rede de apoio social e afetivo dos participantes e para a promoção da saúde psicológica dos mesmos, aumentando diversos fatores de proteção, diminuindo fatores de risco e promovendo a resiliência dos jovens.

Contrariamente, quando Zocateli (2010) procurou estudar a correlação entre a frequência de prática esportiva (em clubes, escolas e outros locais) e a resiliência em crianças e jovens portugueses e brasileiros entre os 12 e os 19 anos que frequentavam escolas públicas de ambos os países, não encontrou diferenças entre os que praticavam e os que não o faziam. Ou seja, os resultados não sustentavam uma relação entre prática de esporte e resiliência.

Entretanto, ainda em relação com o conceito de resiliência, considera-se atualmente existirem três conjuntos de fatores implicados no desenvolvimento de comportamentos resilientes: (a) os atributos e características individuais dos sujeitos, (b) aspectos relacionados às famílias, e (c) características dos ambientes sociais com os quais o sujeito 
interage. Este último aspecto promove a discussão sobre a importância do suporte social para o processo da resiliência (Masten \& Garmezy, 1985; Werner \& Smith, 1992). Pode-se definir suporte social como a existência ou a disponibilidade de pessoas em quem se pode confiar, pessoas com as quais podemos contar para nos ajudar, que se preocupam conosco e que nos dão valor. O suporte social constitui-se então como um mecanismo de proteção para o enfrentamento do risco e, como tal, contribui efetivamente para o desenvolvimento de comportamentos resilientes.

Alguns autores consideram a existência de quatro fatores principais de proteção: (a) relações efetivas e significativas com adultos que possam oferecer apoio, incentivo e orientação, (b) estabelecimento de relações saudáveis com o grupo de pares, (c) desenvolvimento de estratégias eficazes nas soluções de problemas, e (d) inserção ou acesso a comunidades que se preocupam com o bem comum de seus membros (Dumont \& Provost, 1999; Grotberg, 2001; Tiet et al., 1998).

Hoje em dia, a suposição de que o apoio social contribui para um melhor e mais positivo ajuste no desenvolvimento social, fornecendo uma proteção efetiva para os efeitos do estresse e para redução dos danos potenciais das situações de risco é comumente aceita pelos pesquisadores da área (Sarason, Levine, Basham, \& Sarason, 1983), existindo já diversas pesquisas que comprovam o seu papel determinante a esse nível. March (2004), por exemplo, realizou um estudo visando compreender as relações entre a resiliência e as adversidades da vida e sugeriu que a resiliência, por si, só não poderá explicar em sua totalidade o bem-estar subjetivo na idade adulta, destacando o suporte social como uma importante variável a ser considerada. Além disso, recorde-se o anteriormente mencionado estudo de Galli e Vealey (2008), no qual o suporte social emergiu como determinante no desenvolvimento de comportamentos resilientes. Na mesma linha, nos anos mais recentes foram surgindo diversas pesquisas que concluíram sobre a importância da percepção do suporte social fornecido por vários agentes (família, treinadores, colegas de equipe), afirmando que este constitui um fator de proteção de situações adversas e de estresse, promovendo o sucesso e a resiliência dos atletas (Fletcher \& Sarkar, 2012; Howells \& Fletcher, 2015; White \& Bennie, 2015).

Em suma, parece ser inegável que uma estrutura mental resiliente e uma rede de suporte social são essenciais para lidar com situações adversas, existindo diversas pesquisas que comprovam essa importância no esporte de competição. Todavia, não existem muitos estudos desenvolvidos com o objetivo de conhecer o nível de resiliência dos praticantes de esportes comuns, ou seja, aqueles que não participam em atividades esportivas de competição e que apenas dedicam um período do seu tempo para a prática esportiva quer seja por prazer, para manterem um corpo mais saudável, e/ou por outras razões que vão além da prática esportiva competitiva. Assim, carecemos de estudos que possam demonstrar esta relação entre resiliência e esporte em sua concretude, e que também analisem o papel e importância do suporte social enquanto fator de proteção na vida das crianças e jovens.

Assim, a presente pesquisa procurou analisar os níveis de prática esportiva, resiliência e o suporte social de crianças e jovens de escolas públicas brasileiras, examinando, num primeiro momento, as relações entre estas variáveis e o sexo e a idade. Num segundo momento, procuramos verificar se existiam diferenças nos níveis de resiliência e suporte social em função dos níveis de prática esportiva. Acreditamos que esta pesquisa poderá ajudar a compreender melhor a relação entre resiliência, suporte social e prática esportiva e 
a pensar que intervenções são mais eficazes, eventualmente ajudando a desenvolver intervenções específicas e mais apropriadas às necessidades dos dois sexos e a crianças e jovens em diferentes estágios de desenvolvimento.

\section{Método}

\section{Participantes}

Participaram nesta pesquisa 1052 alunos (59.3\% do sexo feminino e $40.7 \%$ do sexo masculino), com idades compreendidas entre os 12 e os 20 anos $(15,53 \pm 1,68)$. Todos os alunos frequentavam escolas públicas do ensino médio situadas em periferias de duas grandes cidades brasileiras.

\section{Instrumentos}

Os participantes preencheram uma bateria de questionários que, para além de variáveis sociodemográficas (idade, sexo), incluía medidas de avaliação da resiliência, suporte social e frequência de prática esportiva. Estes instrumentos são descritos de seguida.

Escala de Resiliência (ER): Trata-se da versão traduzida e adaptada à realidade brasileira (Pesce et al., 2005) da Resilience Scale, desenvolvida originalmente por Wagnild e Young (1993). Este instrumento compreende 25 itens divididos por cinco subescalas com cinco itens cada: equanimidade, perseverança, auto-suficiência, sentido da vida e singularidade existencial. Os sujeitos devem indicar o seu grau de acordo com cada afirmação, numa escala tipo Likert que varia entre 1 (Discordo plenamente) e 7 (Concordo plenamente). Um score total de resiliência pode obtido calculando-se a média das respostas a todos os itens. Wagnild e Young (1993) apresentaram dados que suportam a fiabilidade e validade concorrente do instrumento.

Escala Reduzida de Suporte Social (ERSS): A ERSS é a versão traduzida e adaptada à realidade brasileira (Castelar-Perim, Fonseca, Corte-real \& Dias, 2011) do Social Support Questionnaire - Short Form (SSQ6; Sarason, Sarason, Shearin, \& Pierce, 1987) e avalia o número de pessoas que compõe a rede social do sujeito (fator SSQ6N) e o seu grau de satisfação com o suporte social percebido (SSQ6S). Primeiro os sujeitos devem indicar, relativamente a seis itens distintos (por exemplo: "Quem o aceita incondicionalmente, incluindo as coisas piores e as melhores?"), os elementos que lhe providenciam ajuda ou apoio social, indicando as iniciais dessa pessoa (uma por item) e a sua relação com ela, podendo optar por colocar no máximo 9 pessoas ou escolher ainda como opção colocar "ninguém". Posteriormente, devem assinalar, relativamente aos mesmos itens, o seu grau de satisfação ("Até que ponto está satisfeito com esse apoio?"); para tal devem recorrer a uma escala tipo Likert entre 1 (Muito Insatisfeito) e 6 (Muito Satisfeito). De acordo com os seus autores, a SSQ6 possui uma elevada fiabilidade interna e elevadas correlações com a versão original do Social Support Questionnaire (Sarason et al., 1983) e com outras medidas de personalidade (Sarason et al., 1987).

Atividade esportiva: O questionário que avaliava a frequência de prática esportiva foi desenvolvido especificamente para este estudo e questionava os sujeitos quanto aos seus hábitos de frequência semanal de prática esportiva fora da escola (nunca, até 1 vez/ semana, 2 / 3 vezes/ semana, mais de 3 vezes/ semana). 


\section{Procedimentos de coleta de dados}

Os participantes foram escolhidos utilizando um único critério: serem alunos de escolas públicas de periferias, em escolas de ensino médio. Inicialmente foi obtida a anuência da direção, dos professores, dos pais e dos alunos, em colaborarem com a pesquisa; após obtenção destas devidas autorizações e de garantido o anonimato dos participantes, os questionários foram aplicados por um dos pesquisadores, durante o tempo de uma aula (50 minutos). A participação era facultativa.

\section{Procedimentos de tratamento dos dados}

No que diz respeito à prática esportiva, os participantes foram divididos em três grupos: (a) "não praticantes" (29.1\%), que não praticavam esportes: (b) "praticantes regulares" (34\%), que praticavam entre uma e duas vezes por semana, e (c) "praticantes muito regulares" (36.9\%), que praticavam pelo menos três vezes por semana. Cabe esclarecer que, numa das escolas pesquisadas, não existia quadra esportiva e/ ou outro espaço onde as aulas de Educação Física fossem ministradas. Por isso, foi possível, mesmo tendo coletado os dados no ambiente escolar, conseguir um número numericamente razoável de não praticantes. Considerando o escalão etário, os sujeitos foram distribuídos por três categorias: (a) "adolescência inicial", compreendo os alunos até 15 anos; (b) "adolescência", com alunos entre os 15 e os 17; e (c) "jovens adultos", incluindo alunos entre os 18 e os 20 anos.

Os dados foram analisados por meio do Statistical Package for the Social Sciences (SPSS, versão 17), tendo sido utilizadas estatísticas descritivas (frequências, médias e desvio padrão), a correlação de Pearson (para analisar a relação entre idade e resiliência, bem como as intercorrelações entre as subescalas da resiliência), e técnicas de comparação entre grupos: (a) teste do qui-quadrado para comparação de níveis de prática esportiva em função do sexo e idade; (b) teste t de Student para comparação da resiliência e suporte social em função do sexo; e (c) ANOVA para comparação da resiliência e suporte social em função da faixa etária e do nível de prática esportiva.

\section{Resultados e discussão}

\section{Prática esportiva, resiliência e suporte social: análise em função do sexo e da idade}

A análise dos dados relativos à frequência de prática esportiva em função do sexo revelou diferenças estatisticamente significativas $\left(X^{2}=53.76, p<.001\right)$, verificando-se que os rapazes praticavam esportes com mais regularidade do que as raparigas. Do mesmo modo, ao analisarmos os dados em função da idade também verificamos a existência de diferenças significativas $\left(X^{2}=48.58, p<.001\right)$. Neste caso, os mais jovens, no início da adolescência, praticavam esporte com mais regularidade que os jovens adultos. Estes resultados estão de acordo com os resultados de outros estudos com populações semelhantes, os quais mostram que, de um modo geral, há uma prevalência de praticantes do sexo masculino e sugerem existir uma diminuição de prática com o avançar da idade, registrando-se declínios mais acentuados no final da adolescência (Balaguer \& Castillo, 2002; Corte-Real, 2006; Matos, Simões, Carvalhosa, Reis, \& Canha, 2003).

Quando comparamos os níveis de resiliência em função do sexo, não encontramos diferenças significativas. Relativamente à comparação em função da idade, verificamos que, 
com exceção da singularidade existencial, havia diferenças em todas as subescalas. De um modo geral, os alunos mais velhos exibiam níveis de resiliência mais elevados que os mais novos, sendo as diferenças significativas entre os alunos no início da adolescência e os jovens adultos (Tabela 1).

Tabela 1 - Escores de resiliência. Análise em função da faixa etária

\begin{tabular}{|c|c|c|c|c|c|}
\hline & $\begin{array}{c}\text { Adolescência } \\
\text { inicial } \\
(n=235) \\
\end{array}$ & $\begin{array}{l}\text { Adolescência } \\
\qquad(n=698)\end{array}$ & $\begin{array}{l}\text { Jovens Adultos } \\
\qquad(n=119)\end{array}$ & Anova & Scheffé \\
\hline & $M$ & $M$ & $M$ & & \\
\hline Auto-suficiência & 4.93 & 5.14 & 5.34 & $F=5.45 ; p=.004$ & $\begin{array}{l}\text { I vs II } p<.05 \\
\text { I vs III } p<.01 \\
\text { II vs III } p=.24\end{array}$ \\
\hline Sentido da vida & 5.01 & 5.46 & 5.24 & $F=18.42 ; p<.001$ & $\begin{array}{l}\text { I vs II } p<.001 \\
\text { I vs III } p=.11 \\
\text { II vs III } p=.09\end{array}$ \\
\hline Equanimidade & 4.33 & 4.67 & 4.40 & $F=11.05 ; p<.001$ & $\begin{array}{l}\text { I vs II } p<.001 \\
\text { I vs III } p=.87 \\
\text { II vs III } p<.05\end{array}$ \\
\hline Perseverança & 5.10 & 5.53 & 5.50 & $F=14.56 ; p<.001$ & $\begin{array}{l}\text { I vs II } p<.001 \\
\text { I vs III } p<.05 \\
\text { II vs III } p=.93\end{array}$ \\
\hline $\begin{array}{l}\text { Significado } \\
\text { Existencial }\end{array}$ & 5.00 & 5.01 & 5.14 & $F=0.94 ; p=.401$ & $\begin{array}{l}\text { I vs II } p=.87 \\
\text { I vs III } p=.40 \\
\text { II vs III } p=.53\end{array}$ \\
\hline $\begin{array}{l}\text { Resiliência } \\
\text { Total }\end{array}$ & 4.87 & 5.16 & 5.12 & $F=10.87 ; p=.004$ & $\begin{array}{l}\text { I vs II } p<.001 \\
\text { I vs III } p<.05 \\
\text { II vs III } p=.90\end{array}$ \\
\hline
\end{tabular}

De seguida, procurando aprofundar estes resultados, procedemos à análise das correlações entre a resiliência e a idade. Estas análises demonstraram a existência de correlações positivas entre a idade e algumas subescalas de resiliência, nomeadamente a autossuficiência, sentido da vida, perseverança e o escore total. Além disso, as diferentes subescalas da resiliência também se inter-relacionavam positivamente entre si (Tabela 2).

Tabela 2 - Correlações entre a idade e a resiliência

\begin{tabular}{|c|c|c|c|c|c|c|}
\hline & Idade & $\begin{array}{c}\text { Auto- } \\
\text { suficiência }\end{array}$ & $\begin{array}{l}\text { Sentido } \\
\text { da vida }\end{array}$ & Equanimidade & Perseverança & $\begin{array}{c}\text { Singularidade } \\
\text { existencial }\end{array}$ \\
\hline Idade & - & & & & & \\
\hline Auto-suficiência & $.09 *$ & - & & & & \\
\hline Sentido da vida & $.12^{*}$ & $.50 * *$ & - & & & \\
\hline Equanimidade & .05 & $.38 * *$ & $.41^{* *}$ & - & & \\
\hline Perseverança & $.13^{* *}$ & $.60 * *$ & $.66^{* *}$ & $.36 * *$ & - & \\
\hline $\begin{array}{l}\text { Singularidade } \\
\text { existencial }\end{array}$ & .02 & $.57^{* *}$ & $.55^{* *}$ & $.45^{* *}$ & $.51^{* *}$ & - \\
\hline Resiliência total & $.10^{*}$ & $.80 * *$ & $.80 * *$ & $.66^{* *}$ & $.81^{* *}$ & $.81^{* *}$ \\
\hline \multicolumn{7}{|c|}{$* * p<.01 ; * * * p<.001$} \\
\hline
\end{tabular}


Os resultados relativos às diferenças entre sexos estão de acordo com a revisão que Wagnild (2009) fez dos estudos que tinham utilizado a Resilience Scale, na qual o autor apenas encontrou diferenças em dois dos doze estudos analisados (favorecendo os homens). Por outro lado, contrariam as conclusões do mesmo autor com relação à variável idade, uma vez que, a este respeito, nenhum dos estudos mostrou diferenças significativas. Entretanto, autores como Lundman et al. (2007) encontraram relações positivas entre a idade e resiliência, justificando estes dados a partir da análise da resiliência como um processo que ocorre durante toda a vida das pessoas. Este aspecto é também teoricamente defendido por outros pesquisadores (ver: Galli \& Vealey, 2008; Luthar et al., 2000; Rutter, 2006).

No que se refere ao suporte social, não foram encontradas diferenças significativas número de pessoas que os sujeitos dos dois sexos percebiam como compondo as suas redes de apoio social. Quanto ao grau de satisfação dos sujeitos com essa mesma rede de apoio, as diferenças eram significativas e favoráveis às meninas. A respeito da relação entre a idade e o suporte social, também só existiam diferenças significativas no grau de satisfação sendo este mais elevado nos alunos incluídos no grupo da adolescência do que naqueles no início da adolescência (Tabela 3).

Tabela 3 - Escores alcançados no SSQ6. Análise em função da faixa etária

\begin{tabular}{|c|c|c|c|c|c|}
\hline & $\begin{array}{c}\text { Adolescência } \\
\text { inicial } \\
(n=188) \\
\end{array}$ & $\begin{array}{l}\text { Adolescência } \\
\qquad(n=628)\end{array}$ & $\begin{array}{l}\text { Jovens Adultos } \\
\qquad(n=102)\end{array}$ & Anova & Scheffé \\
\hline & $M$ & $M$ & $M$ & & \\
\hline $\begin{array}{l}\text { № de pessoas na } \\
\text { rede de apoio }\end{array}$ & $19.60 \pm$ & $19.88 \pm$ & $20.00 \pm$ & $F=0.07 ; p=.93$ & $\begin{array}{ll}\text { I vs II } & p<.94 \\
\text { I vs III } & p<.95 \\
\text { I| vs III } & p=.99\end{array}$ \\
\hline $\begin{array}{l}\text { Satisfação com } \\
\text { rede de apoio }\end{array}$ & $27.13 \pm$ & $29.25 \pm$ & $27.55 \pm$ & $F=4.22 ; p=.02$ & $\begin{array}{ll}\text { I vs II } & p<.05 \\
\text { I vs III } & p=.94 \\
\text { II vs III } & p=.25\end{array}$ \\
\hline
\end{tabular}

Os resultados da comparação entre sexos concordam com pesquisas anteriores que sugerem diferenças nas fontes, tipo e no modo como homens e mulheres percebem o suporte social (Stuntz, Sayles, \& McDermott, 2011; Wigglesworth, Young, Medic, \& Grovec, 2012). Na nossa pesquisa, os resultados sugerem que, mesmo tendo um número de componentes de suas redes de apoio muito semelhante, as moças estavam mais satisfeitas com essas redes do que os rapazes. Leung, Chen, Lue e Hsu (2007) sugeriram que, em uma rede de suporte social forte, o mais importante não é o número de contatos, mas sim a qualidade dos relacionamentos que a compõem.

As diferenças encontradas entre os alunos na adolescência inicial e na adolescência no que diz respeito à satisfação com a sua rede de apoio social são consonantes com estudos prévios, indicando que, em diferentes estágios de desenvolvimento, os sujeitos têm diferentes necessidades de suporte social ao longo do seu desenvolvimento (Lauer et al., 2010; Voorhees et al., 2005). Assim, estes dados podem ser um indicador de um certo desenvolvimento de autonomia, uma vez que, com a maturidade, existe uma tendência para 
se valorar mais a qualidade das relações do q que a sua quantidade. Uma outra explicação pode estar relacionada ao desenvolvimento da resiliência.

Com efeito, um dos construtos que constitui a resiliência é a singularidade existencial (pelo menos o que é expresso na escala de resiliência de Wagnild e Young, 1993). Isso sugere que o sujeito se percebe como único, que não só é capaz de resolver as suas questões, como percebe ser necessário resolvê-las por si só. Ora, este sentimento poderia explicar uma maior satisfação com o suporte social, ainda que com um número relativamente baixo de componentes das redes de suporte social. Por outro lado, quando comparados os resultados dos adolescentes e dos jovens adultos, tal fato não ocorreu, o que põe em causa o que referimos anteriormente, sugerindo antes a necessidade do desenvolvimento de mais estudos que possam esclarecer este fato.

\section{Relação entre resiliência, suporte social e prática esportiva}

Para procedermos à análise das relações entre a prática esportiva e a resiliência recorremos às três categorias de prática esportiva mencionadas anteriormente: não praticantes, praticantes regulares e praticantes muito regulares. Nos resultados apresentados na Tabela 4 pode-se notar que, excetuando a diferença entre os não praticantes e os praticantes muito regulares na subescala de sentido da vida, todas as restantes diferenças foram estatisticamente significativas. Como era esperado, os praticantes muito regulares exibiam melhores índices de resiliência que os não praticantes. Todavia, encontrou-se também um dado curioso, pois os praticantes regulares exibiam consistentemente níveis mais baixos de resiliência do que os não praticantes.

Tabela 4 - Escores de resiliência. Análise em função da prática esportiva

\begin{tabular}{|c|c|c|c|c|c|}
\hline & $\begin{array}{c}\text { Não } \\
\text { praticantes } \\
(n=306)\end{array}$ & $\begin{array}{l}\text { Praticantes } \\
\text { regulares } \\
(n=358)\end{array}$ & $\begin{array}{c}\text { Praticantes muito } \\
\text { regulares } \\
(n=388)\end{array}$ & Anova & Scheffé \\
\hline & $M$ & $M$ & $M$ & & \\
\hline Auto-suficiência & 5.08 & 4.86 & 5.38 & $F=20.25 ; p=.001$ & $\begin{array}{c}\text { I vs II } p<.05 \\
\text { I vs III } p<.01 \\
\text { II vs III } p=.001\end{array}$ \\
\hline Sentido da vida & 5.44 & 5.07 & 5.50 & $F=19.58 ; p<.001$ & $\begin{array}{c}\text { I vs II } p<.001 \\
\text { I vs III } p=.78 \\
\text { II vs III } p=.001\end{array}$ \\
\hline Equanimidade & 4.57 & 4.29 & 4.80 & $F=24.34 ; p<.001$ & $\begin{array}{c}\text { I vs II } p<.001 \\
\text { I vs III } p=.05 \\
\text { II vs III } p<.001\end{array}$ \\
\hline Perseverança & 5.44 & 5.15 & 5.68 & $F=22.42 ; p<.001$ & $\begin{array}{c}\text { I vs II } p<.001 \\
\text { I vs III } p<.05 \\
\text { II vs III } p<.001\end{array}$ \\
\hline $\begin{array}{l}\text { Singularidade } \\
\text { Existencial }\end{array}$ & 5.02 & 4.68 & 5.32 & $F=27.03 ; p<.001$ & $\begin{array}{c}\text { I vs II } p<.001 \\
\text { I vs III } p<.01 \\
\text { II vs III } p=.001\end{array}$ \\
\hline $\begin{array}{l}\text { Resiliência } \\
\text { Total }\end{array}$ & 5.11 & 4.81 & 5.33 & $F=37.96 ; p<.001$ & $\begin{array}{c}\text { I vs II } p<.001 \\
\text { I vs III } p<.01 \\
\text { II vs III } p=.001\end{array}$ \\
\hline
\end{tabular}


Na revisão da literatura não encontramos consistência nas conclusões em relação à prática esportiva e resiliência. Por um lado, estudos realizados com atletas (Galli \& Vealey, 2008; Hosseini \& Besharat, 2010) encontraram uma correlação positiva entre a prática esportiva e ganhos no desenvolvimento de resiliência. Por outro lado, em contextos não competitivos há resultados díspares: Zocateli (2010) não encontrou diferenças estatisticamente significativas que pudessem sustentar esta relação positiva entre prática esportiva e resiliência, mas Sanches (2007) verificou efeitos positivos ao nível da resiliência em jovens envolvidos num programa de atletismo. Os resultados encontrados na nossa pesquisa reforçam a ideia que, de fato, a prática esportiva possui uma correlação positiva com a resiliência. Porém, os níveis de resiliência mais elevados nos não praticantes, em comparação com os praticantes regulares, sugerem que será necessária uma prática muito regular para esses efeitos se fazerem sentir. No entanto, vale sublinhar que, neste trabalho, a amostra se constituiu exclusivamente de não atletas.

Ao comparar o suporte social em função da prática esportiva, verificou-se que os que praticantes regulares reportavam um número mais elevado de sujeitos na sua rede social de apoio que os não praticantes. Além disso, os que praticavam esporte muito regularmente estavam mais satisfeitos com a sua rede de apoio do que os não praticantes ou os praticantes regulares (Tabela 5).

Tabela 5 - Escores no SSQ6 entre as três categorias da prática esportiva

\begin{tabular}{|c|c|c|c|c|c|}
\hline & $\begin{array}{l}\text { Não praticantes } \\
\quad(n=264)\end{array}$ & $\begin{array}{c}\text { Praticantes } \\
\text { regulares } \\
(n=308) \\
\end{array}$ & $\begin{array}{c}\text { Praticantes } \\
\text { muito regulares } \\
(n=346)\end{array}$ & Anova & Scheffé \\
\hline & $M$ & $M$ & $M$ & & \\
\hline $\begin{array}{l}\text { № de pessoas na } \\
\text { rede de apoio }\end{array}$ & 18.44 & 21.21 & 19.67 & $F=5.32 ; p=.005$ & $\begin{array}{l}\text { I vs II } p<.01 \\
\text { I vs III } p=.34 \\
\text { II vs III } p=.15\end{array}$ \\
\hline $\begin{array}{l}\text { Satisfação com } \\
\text { rede de apoio }\end{array}$ & 28.03 & 27.58 & 30.01 & $F=5.9 ; p=.003$ & $\begin{array}{l}\text { I vs II } p=.85 \\
\text { I vs III } p<.05 \\
\text { II vs III } \quad p<.01\end{array}$ \\
\hline
\end{tabular}

Estes dados sugerem que a uma prática esportiva muito regular está associada uma maior satisfação com as redes de apoio, independentemente do número de pessoas que as compõem. Pesquisas anteriores que revisaram os correlatos de atividade física em adolescentes concluíram que o suporte social de família e amigos estava associado a níveis mais elevados de prática (Biddle, Whitehead, O’Donovan \& Nevill, 2005; Lubans \& Morgan, 2009). Se tivermos em conta que a satisfação com a rede de apoio sugere uma boa percepção, por parte do sujeito, de que se trata de uma rede eficaz, à qual ele pode recorrer quando precisar podemos sugerir que esta percepção estará associada à qualidade dos vínculos que são estabelecidos. Recordemos que um dos benefícios importantes que a prática esportiva pode oferecer é a existência de uma boa relação no grupo de pares e com adultos significativos (Brodkin \& Weiss, 1990; Weiss \& Petlichkoff, 1989). 


\section{Considerações finais}

Esta pesquisa procurou analisar a relação entre os níveis de prática esportiva, resiliência e suporte social de crianças e jovens de escolas públicas brasileiras, examinando a existência de diferenças em alunos dos dois sexos e em diferentes faixas etárias; foram também analisadas diferenças na resiliência e suporte social em função do nível de prática esportiva.

Por um lado, ao mostrarem que os rapazes e os alunos mais novos estavam menos satisfeitos com o suporte social recebido, e que os jovens adultos eram mais resilientes que aqueles no início da adolescência, os resultados sugerem a importância de providenciar suporte social diferenciado aos diferentes sexos, tendo também atenção ao estágio de desenvolvimento/ idade em que se encontram. Vale salientar que, no caso do suporte social, esta individualização parece ser independente do número de pessoas que constituem a rede se suporte social, sendo mais importante a qualidade do que a quantidade.

Adicionalmente, de um modo global, os resultados indicaram a existência de associações positivas entre a prática esportiva e a resiliência, parecendo confirmar, uma vez mais, a importância da primeira no desenvolvimento da segunda. Contudo, não podemos deixar de reconhecer que alguns resultados não eram esperados, como os que respeitam aos níveis de resiliência mais elevados nos não praticantes, em comparação com os praticantes regulares. Embora, num primeiro momento, pareçam sugerir que será necessária uma prática muito regular para os efeitos se fazerem sentir ao nível da resiliência, pensamos que são necessárias mais pesquisas que confirmem ou infirmem esta relação.

Por outro lado, salientando que nesta pesquisa os participantes eram exclusivamente não atletas, parece-nos relevante salientar a importância de se buscar compreender o sentido da correlação entre prática esportiva e resiliência. Com efeito, nesta pesquisa estabelecemos que existe esta correlação, mas é preciso estudar as relações que a estabelecem. Considerando que diversas pesquisas no âmbito da psicologia do esporte, com atletas de competição, mostraram que as características da prática esportiva parecem favorecer o desenvolvimento da resiliência, nos parece bastante relevante que se busque aprofundar cada vez mais esta relação, também em contextos não-competitivos. Afinal o que favorece o quê? Será a prática esportiva que contribui para o desenvolvimento da resiliência ou será que os sujeitos, por serem resilientes, praticam mais esportes? Ou se trata de uma relação de fato dialética?

A resposta a estas e outras questões pode ser encontrada quer em pesquisas de caráter mais qualitativo, que possam buscar o sentido, a direção desta relação, mas também em pesquisas quantitativas que incluam delineamentos longitudinais ou experimentais que permitam a inferência de relações causa-efeito e/ ou que incluam, por exemplo, o suporte social como variável de controle em modelos de equação estrutural ou regressões lineares. Ou seja, nos parece ser bastante pertinente que novos estudos possam contribuir para o desenvolvimento do conhecimento das relações entre a prática esportiva, a resiliência e o suporte social, não só entre atletas de elite mas também tendo como participantes crianças, jovens e adultos praticantes amadores, a fim de podermos compreender cada vez mais as possibilidades que esta prática pode oferecer para o desenvolvimento da resiliência numa sociedade cada vez mais chamada ao enfrentamento de grandes riscos e dificuldades. Todavia, enquanto não temos resposta definitiva a esta questão, todos os dados, incluindo os da nossa pesquisa, nos habilitam a sugerir que os efeitos positivos da atividade física, exercício e esporte, em termos físicos e psicológicos, incluindo ao nível da resiliência, 
sugerem a importância da promoção de estilos de vida ativos, em qualquer população e em qualquer idade.

\section{Referências}

Assis, S. G., Avanci, J. Q., Pesce, R. P., \& Deslandes, S. F. (2006). Superação de dificuldades na infância e adolescência: Conversando com profissionais de saúde sobre resiliência e promoção da saúde. Rio de Janeiro, Brasil: FIOCRUZ/ ENSP/ CLAVES/ CNPq.

Balaguer, I. (2000). Un estudio sobre los predictores de los estilos de vida saludables de los adolescentes valencianos. Valência: Dirección General de la Salut Pública, Conselleria de Sanitat.

Balaguer, I., \& Castillo, I. (2002). Actividad física, ejercicio físico y deporte en la adolescencia temprana. In I. Balaguer (Org.). Estilos de vida en la adolescência (pp. 37-64). Valencia: Promolibro.

Beeghly, M., \& Cicchetti, D. (1994). Child maltreatment, attachment, and the self-system: Emergence of an internal state lexicon in toddlers at high social risk. Development and Psychopathology, 6(1), 5-30.

Biddle, S. J., Whitehead, S. H., O’Donovan, T. M., \& Nevill, M. E. (2005). Correlates of participation in physical activity for adolescent girls: a systematic review of recent literature. Journal of Physical Activity \& Health, 2(4), 423.

Brodkin, P., \& Weiss, M. R. (1990). Developmental differences in motivation for participating in competitive swimming. Journal of Sport and Exercise Psychology, 12(2), 248-263.

Brustad, R. (1988). Affective outcomes in competitive youth sport: The influence of intrapersonal and socialization factors. Journal of Sport and Exercise Psychology, 10, 307-321.

Castelar-Perim, P., Fonseca, A. M., Corte-real, N., \& Dias, C. (2011). Resiliência e prática desportiva: Um estudo realizado com adolescentes brasileiros. Tese de Doutorado, Facudade de Desporto, Universidade do Porto, Porto, Portugal.

Cicchetti, D., \& Rogosch, F. A. (1997). The role of self-organization in the promotion of resilience in maltreated children. Development and Psychopathology, 9, 799-817.

Chung, P. K., Chengb, K. C., Li, H. Y., Jiangb, X., Su, N., Zhang, C., \& Si, G. (2013). The effect of resilience training for Hong Kong junior athletes. International Journal of Sport and Exercise Psychology, 11(2), 228-242.

Corte-Real, N. (2006). Desporto, saúde e estilos de vida... Diferentes olhares, objetivos e subjetivos, sobre os comportamentos dos adolescentes. Tese de Doutorado, Faculdade de Ciências do Desporto e de Educação Física, Universidade do Porto, Porto, Portugal.

Cyrulnik, B. (2001). Uma infelicidade maravilhosa. Porto: Ambar.

Dias, C., Corte-Real, N., Corredeira, R., Barreiros, A., Bastos, T., \& Fonseca, A. M. (2008). A prática desportiva dos estudantes universitários e suas relações com as autopercepções físicas, bem-estar subjectivo e felicidade. Estudos de Psicologia, 13(3), 223-232.

Dumont, M., \& Provost, M. A. (1999). Resilience in adolescents: Protective role of social support, coping strategies, self-esteem, and social activities on experience of stress and depression. Journal of Youth and Adolescence, 28(3), 343-363.

Fletcher, D., \& Sarkar, M. (2012). A grounded theory of psychological resilience in Olympic champions. Psychology of Sport and Exercise, 13, 669-678.

Fox, K. R., \& Corbin, C. B. (1989). The Physical Self-Perception Profile: Development and preliminary validation. Journal of Sport and Exercise Psychology, 11, 408-430.

Galli, N., \& Vealey, R. S. (2008). Bouncing back" from adversity: Athletes' experiences of resilience. The Sport Psychologist, 22, 316-335.

Garmezy, N. (1991). Resilience in children's adaptation to negative life events and stressed environments. Pediatric Annals, 20(9), 459-466.

Grotberg, E. (2001). Resilience programs for children in disaster. Ambulatory Child Health, 7, 75-83. 
Hellison, D. (1985). Goals and strategies for physical education. Champaign, IL: Human Kinetics.

Hellison. D., \& Walsh, D. (2002). Responsability-based youth programs evaluation: Investigating the investigations. Quest, 54, 292-307.

Henley, R., Schweizer, I., Gara, F., \& Vetter, S. (2008). How psychosocial sport \& play programs help youth manage adversity: A review of what we know \& what we should research. International Journal of Psychosocial Rehabilitation, 12(1), 51-58.

Hosseini, S. A., \& Besharat, M. A. (2010). Relation of resilience with sport achievement and mental health in a sample of athletes. Procedia - Social and Behavioral Sciences, 5, 633-663.

Howells, K., \& Fletcher, D. (2015). Sink or swim: Adversity- and growth-related experiences in Olympic swimming champions. Psychology of Sport and Exercise, 16, 37-48.

Hunter, A. J., \& Chandler, G. E. (1999). Adolescent resilience. Image. Journal of Nursing Scholarship, 31(2), 243247.

Lauer, M., Albert, S., Aswani, S., Halpern, B., Campanella, L., \& La Rose, D. (2010). Globalization, Pacific Islands, and the paradox of resilience. Global Environmental Change, 23(1), 40-50. doi:10.1016/j.gloenvcha.2012.10.011.

Leppert, K., Gunzelmann, T., Schumacher, J., Strauss, B., \& Brahler, E. (2005). Resilience as a protective personality characteristic in the elderly. Psychotherapie, Psychosomatik, Medizinishe Psychologie, 55(8), 365-369.

Leung, K. K., Chen, C. Y., Lue, B. H., \& Hsu, S. T. (2007). Social support and family functioning on psychological symptoms in elderly Chinese. Archives of Gerontology and Geriatrics, 44, 203-213.

Lubans, D. R., \& Morgan, P. J. (2009). Social, psychological and behavioural correlates of pedometer step counts in a sample of Australian adolescents. Journal of Science \& Medicine in Sport, 12, 141-147.

Lundman, B., Strandberg, G., Eisemann, M., Gustafson, Y., \& Brulin, C. (2007). Psychometric properties of the Swedish version of the Resilience Scale. Scandinavian Journal of Caring Sciences, 21(2), $229-237$.

Luthar, S. S., Cicchetti, D., \& Becker, B. (2000). The construct of resilience: A critical evaluation and guidelines for future work. Child Development, 71(3), 543-562.

March, M. (2004). Well being of older Australians: The interplay of life adversity and resilience in late life development. Doctoral dissertation, Charles Sturt University, Australia.

Marsh, H. W., \& Craven, R. (1997). Academic self-concept: Beyond the dustbowl. In G. D. Phye (Org.). Handbook of classroom assessment (pp. 131-198). San Diego, CA: Academic Press.

Marsh, H. W., \& Perry, C. (2005). Self-concept contributes to winning gold medals: Causal ordering of selfconcept and elite swimming performance. Journal of Sport and Exercise Psychology, 27, 71-91.

Masten, A., \& Coatsworth, J. D. (1998). The development of competence in favorable and unfavorable environments: Lessons from research on successful children. American Psychologist, 53, 205-220.

Masten, A. S., \& Garmezy, N. (1985). Risk, vulnerability, and protective factors in developmental psychopathology. In B. B. Lahey \& A. E. Kazdin (Eds.). Advances in clinical child psychology (pp. 1-52). New York, NY: Plenum Press.

Matos, M., Simões, C., Carvalhosa, S., Reis, C., \& Canha, L. (2003). A saúde dos adolescentes Portugueses: 4 anos depois. Lisboa: Fundação para a Ciência e Tecnologia e Faculdade de Motricidade Humana.

Miller, P. S., \& Kerr, G. (2002). The athletic, academic and social experiences of intercollegiate studentathletes. Journal of Sport Behavior, 25, 346-367.

O'Dougherty-Wright, M., Masten, A. S., Northwood, A., \& Hubbard, J. J. (1997). Long-term effects of massive trauma: Developmental and psychobiological perspectives. In D. Cicchetti \& S. L. Toth (Eds.), Rochester Symposium on Developmental Psychopathology (Vol. 8): Developmental perspectives on trauma (pp. 181-225). Rochester, NY: University of Rochester Press.

O'Dougherty, M., \& Wright, F. S. (1990). Children born at medical risk: Factors affecting vulnerability and resilience. In J. Rolf, A. S. Masten, D. Cicchetti, K. H. Nuechterlein \& S. Weintraub (Eds.). Risk and 
protective factors in the development of psychopathology (pp. 120-140). New York, NY: Cambridge University Press.

Pesce, R. P., Assis, S., Avanci, J. Q., Santos, N. C., Malaquias, J. V., \& Carvalhaes, R. (2005). Adaptação transcultural, confiabilidade e validade da escala de resiliência. Cadernos de Saúde Pública, 21(2), 436448.

Ralha-Simões, H. (2001). Resiliência e desenvolvimento pessoal. In J. Tavares (Org.). Resiliência e educação (pp. 95-114). São Paulo: Cortez.

Regueiras, M. L. V. (2012). Desenvolvimento da responsabilidade pessoal e social dos jovens através do desporto: Uma análise centrada na perspetiva dos especialistas. Tese de Doutorado, Facudade de Desporto, Universidade do Porto, Porto, Portugal.

Richardson, G. E. (2002). The metatheory of resilience and resiliency. Journal of Clinical Psychology, 58(3), 307321.

Richters J. E., \& Martinez, P. (1993). The NIMH community violence project: I. Children as victims of and witnesses to violence. Psychiatry, 56, 7-21.

Rutter, M. (2006). The promotion of resilience in the face of adversity. In A. Clarke \& J. S. Dunn (Eds.). Families count: effects on child and adolescent development (pp. 26-52). New York, NY: Cambridge University Press.

Sanches, S. M. (2007). A prática esportiva como uma atividade potencialmente promotora de resiliência. Revista Brasileira de Psicologia do Esporte, 1(1), 1-15.

Sarason, I. G., Levine, H., Basham, R., \& Sarason, B. (1983). Assessing social support: The Social Support Questionnaire. Journal of Personality and Social Psychology, 44, 127-139.

Sarason, I. G., Sarason, B. R., Shearin, E. N., \& Pierce, G. R. (1987). A brief measure of social support: Practical and theoretical implications. Journal of Social and Personal Relationships, 4, 497-510.

Standage, M., Duda, J. L., \& Pensgaard, A. M. (2005). The effect of competitive outcome and task-involving, ego-involving, and cooperative structures on the psychological well-being of individuals engaged in a coordination task: A self-determination approach. Motivation and Emotion, 29(1), 41-68.

Stuntz, C. P., Sayles, J., \& McDermott, E. (2011). Same-sex and coed sport teams: How the social environment relates to sources of social support and perceived competence. Journal of Sport Behavior, 34, 98-120.

Tiet, Q. Q., Bird, H. R., Davies, M., Hoven, C., Cohen, P., Jensen, P. S., \& Goodman, S. (1998). Adverse life events and resilience. American Academy of Child and Adolescent Psychiatry, 37(11), 1191-1200.

Voorhees, C. C., Murray, D., Welk, G., Birnbaum, A., Ribisl, K. M., Johnson, C. C., \& Jobe, J. B. (2005). The role of peer social network factors and physical activity in adolescent girls. American Journal of Health Behavior, 29, 183-190. doi:10.5993/AJHB.29.2.9.

Wagnild, G. (2009). The Resilience Scale user's guide for the US English version of the Resilience Scale and the 14-Item Resilience Scale (RS-14). Worden, MT: The Resilience Center.

Wagnild, G., \& Young H. M. (1993). Development and psychometric evaluation of the Resilience Scale. Journal of Nursing Measuring, 1, 165-177.

Weiss, M., \& Petlichkoff, L. (1989). Children's motivation for participation in and withdrawal from sport: Identifying the missing links. Pediatric Exercise Science, 1, 195-211.

Wells, R. D., \& Schwebel, A. I. (1987). Chronically ill children and their mothers: Predictors of resilience/ vulnerability to hospital and surgical stress. Journal of Developmental \& Behavioral Pediatrics, 8, 83-89.

Werner, E. E., \& Smith, R. S. (1992). Overcoming the odds: High-risk children from birth to adulthood. Ithaca, NY: Cornell University Press.

White, R. L., \& Bennie, A. (2015). Resilience in youth sport: A qualitative investigation of gymnastics coach and athlete perceptions. International Journal of Sports Science \& Coaching, 10(2+3), 379-393.

Wigglesworth, J. C., Young, B. W., Medic, N., \& Grovec, J. R. (2012). Examining gender differences in the determinants of masters swimmers' sport commitment. International Journal of Sport and Exercise Psychology, 10(3), 236-250. 
Yunes, M. A. M., \& Szymanski, H. (2001). Resiliência: Noção, conceitos afins e considerações críticas. In J. Tavares (Ed.). Resiliência e educação (pp. 13-42). São Paulo, SP: Cortez.

Zocateli, V. (2010). Resiliência e prática desportiva: Um estudo com jovens Brasileiros e Portugueses. Dissertação de Mestrado. Faculdade Desporto, Universidade do Porto, Porto, Portugal.

Zomignani, M. A. (2002). Invulnerável, não: Resiliente. Viver Psicologia, XI(116), 30-31.

Apresentação: 22/02/2015

Aprovação: 22/11/2015 